\title{
D-cycloserine increases the effectiveness of vancomycin against vancomycin-highly resistant Staphylococcus aureus
}

\author{
Fumiaki Tabuchi ${ }^{1}$, Yasuhiko Matsumoto ${ }^{2}$, Masaki Ishii ${ }^{2,3}$, Keita Tatsuno ${ }^{4}$, Mitsuhiro Okazaki ${ }^{5}$, Tomoaki Sato ${ }^{4}$, \\ Kyoji Moriya $^{4}$ and Kazuhisa Sekimizu ${ }^{2,3}$ \\ Vancomycin is a widely used clinical drug to treat for infection by methicillin-resistant Staphylococcus aureus. Some patients \\ show a weak response to vancomycin treatment. We previously reported that $\beta$-lactams increase the susceptibility to vancomycin \\ by vancomycin-highly resistant $S$. aureus (VRSA) strains obtained following repeated in vitro mutagenesis and vancomycin \\ selection. Here we found that the susceptibility of the VRSA strains to vancomycin was remarkably increased by combined \\ treatment with D-cycloserine. On the other hand, VRSA did not show increased susceptibility to vancomycin in combination with \\ bacitracin, fosfomycin, erythromycin, lincomycin, gentamicin, levofloxacin or nisin. Furthermore, in an in vivo infection model \\ with silkworms, combined treatment with vancomycin and D-cycloserine exhibited therapeutic effects, whereas treatment with \\ each compound alone did not. These findings suggest that combined treatment with vancomycin and D-cycloserine could be \\ therapeutically effective against infectious diseases caused by VRSA.
}

The Journal of Antibiotics (2017) 70, 907-910; doi:10.1038/ja.2017.56; published online 7 June 2017

\section{INTRODUCTION}

Vancomycin is clinically used worldwide to treat methicillinresistant Staphylococcus aureus (MRSA) infection. Vancomycinintermediate-resistant $S$. aureus strains, which exhibit weak tolerance against vancomycin (minimum inhibitory concentration (MIC): 4-8 $\mu \mathrm{g} \mathrm{ml}^{-1}$ ), have been clinically isolated. ${ }^{1}$ Vancomycin-highly resistant (VRSA) strains that are highly resistant to vancomycin (MIC: $\geqslant 16 \mu \mathrm{g} \mathrm{ml}^{-1}$ ) have also been reported. ${ }^{2,3}$ Antibiotics such as teicoplanin, linezolid and daptomycin are used for treatment of patients who show a weak response to vancomycin treatment. However, resistant strains to these antibiotics have already been isolated. ${ }^{4-6}$ Each time a novel antibiotic is used clinically to treat infection by vancomycin-resistant strains, resistant strains are expected to emerge. Thus, continual development of novel effective strategies is needed.

We obtained vancomycin-highly resistant ( $\mathrm{MIC} \geqslant 16 \mu \mathrm{g} \mathrm{ml}^{-1}$ ) strains from clinical isolates of MRSA strains by repeating steps of mutagenesis and vancomycin selection. ${ }^{7}$ VRSA strains isolated using this procedure accumulated multiple genetic mutations that led to an increased cell wall thickness and increased tolerance against high concentrations of vancomycin $\left(\geqslant 16 \mu \mathrm{g} \mathrm{ml}^{-1}\right){ }^{7}$ Recently, we found that $\beta$-lactams increased the susceptibility of these strains to vancomycin. ${ }^{7,8}$ In the present study, we searched for other antimicrobial drugs that increased the susceptibility of VRSA to vancomycin.
We found that D-cycloserine decreased the MIC values of vancomycin against VRSA.

The pharmacokinetics and toxicity of drug candidates is difficult to estimate in vitro, because the results often differ from those in vivo. Therefore, animal models are required to evaluate the therapeutic effects of drug candidates. ${ }^{9-12}$ We established a silkworm infection model to evaluate the therapeutic effects of antibacterial and antifungal agents. ${ }^{13-15}$ The silkworm infection model has several advantages compared to mammalian models, including lower breeding costs and fewer ethical problems with regard to animal welfare. The pharmacokinetic parameters, such as the half-life and proteinbinding capacities of antibiotics, are consistent between silkworms and mammals. ${ }^{16}$ Furthermore, the median effective dose $\left(\mathrm{ED}_{50}\right)$ values (mg per gram animal) of antibiotics are also consistent between the silkworm infection model and mammalian models. ${ }^{15,17}$ Using the silkworm infection model, we recently discovered a novel antibiotic, Lysocin E, which was effective against $S$. aureus infection in a mouse model. ${ }^{18}$ Silkworm models are used for the selection of drug candidates through monitoring their therapeutic effectiveness against infectious diseases. ${ }^{19-23}$ We established a silkworm infection model to evaluate the therapeutic effects of combined vancomycin and $\beta$-lactams against VRSA. ${ }^{8}$ In the present study, we used the silkworm infection model to evaluate the therapeutic effects of a combination of vancomycin and D-cycloserine against VRSA.

\footnotetext{
${ }^{1}$ Laboratory of Microbiology, Graduate School of Pharmaceutical Sciences, The University of Tokyo, Tokyo, Japan; ${ }^{2}$ Teikyo University Institute of Medical Mycology, Tokyo, Japan; ${ }^{3}$ Genome Pharmaceuticals Institute Co. Ltd, Tokyo, Japan; ${ }^{4}$ Faculty of Medicine, Department of Infection Control and Prevention, The University of Tokyo, Tokyo, Japan and ${ }^{5}$ Department of Medical Technology, School of Health Sciences, Tokyo University of Technology, Tokyo, Japan

Correspondence: Dr Y Matsumoto or Dr K Sekimizu, Teikyo University Institute of Medical Mycology, 359 Otsuka, Hachioji, Tokyo 192-0395, Japan.

E-mail: ymatsumoto@main.teikyo-u.ac.jp or sekimizu@main.teikyo-u.ac.jp

Received 13 March 2017; revised 10 April 2017; accepted 11 April 2017; published online 7 June 2017
} 
Table 1 MIC values of various antimicrobial drugs against VR7 and MR7

\begin{tabular}{|c|c|c|c|c|c|c|c|c|c|c|}
\hline Strains & VAN & $C S$ & $B C$ & FOM & $E M$ & $L C M$ & $G M$ & $T C$ & LVFX & $N C$ \\
\hline MR7 & 1 & 64 & 64 & $>256$ & $>256$ & $>256$ & 256 & 128 & $>256$ & $>256$ \\
\hline
\end{tabular}

Abbreviations: BC, bacitracin; CS, D-cycloserine; EM, erythromycin; FOM, fosfomycin; GM, gentamicin; LCM, lincomycin; LVFX, levofloxacin; MIC, minimum inhibitory concentration; NC, nisin;

TC, tetracycline; VAN, vancomycin.

MIC values of antimicrobial drugs against VR7 and MR7 were determined by the micro-dilution method. Unit: $\mu \mathrm{g} \mathrm{ml}{ }^{-1}$.

\section{METHODS}

\section{Bacterial strains and culture conditions}

Eight MRSA strains (MR1-8) were isolated from human patients at the University of Tokyo Hospital. ${ }^{7}$ Vancomycin-resistant strains (VR1-8) isolated from MRSA strains (MR1-8) by repeating steps of mutagenesis and antibiotic selection ${ }^{7}$ were used in the experiments. Bacteria were cultured at $37^{\circ} \mathrm{C}$ in Tryptic Soy Broth according to the previously described method. ${ }^{7}$ RN4220 is widely used as a laboratory strain. ${ }^{24}$

\section{Measurement of antibacterial activity}

The MIC values of various antibiotics were determined by the micro-dilution method according to Tabuchi et al. ${ }^{8}$ Briefly, bacterial suspension was dispensed in the wells of a 96-well round-bottom plate. Solutions containing test samples were added, mixed and serially diluted twofold. After $48 \mathrm{~h}$ incubation, bacterial growth in each well was measured as described previously. ${ }^{7}$ A greater than fourfold difference in the MIC values was judged to be significant.

Evaluation of the therapeutic effects of antimicrobial reagents in the silkworm infection model

Evaluation of the therapeutic effects of antibiotic reagents using the silkworm infection model was performed according to Tabuchi et al. ${ }^{8}$ Briefly, $50 \mu \mathrm{l}$ of a twofold diluted overnight culture of $S$. aureus (injected bacterial numbers were $0.45-1.1 \times 10^{8}$ per larva) was injected into the silkworm hemolymph, followed by injection of $50 \mu \mathrm{l}$ of sterilized saline or various concentrations of antibiotics. After injection with the bacterial culture and antibiotics, the silkworms were reared at $37^{\circ} \mathrm{C}$ and silkworm survival was monitored. Survival curves were obtained by five independent experiments. The log-rank test was performed for statistical processing using Prism software (GraphPad Software, La Jolla, CA, USA).

\section{RESULTS}

Search for antimicrobial drugs that increase the susceptibility of VRSA to vancomycin

We previously reported that $\beta$-lactams increased the susceptibility to vancomycin of VRSA strains obtained from MRSA stains by in vitro mutagenesis. ${ }^{8}$ In this study, we searched for other antimicrobial compounds that increased the susceptibility of VRSA to vancomycin. The antimicrobial drugs tested were cell wall synthesis inhibitors: D-cycloserine, bacitracin and fosfomycin; protein synthesis inhibitors erythromycin, lincomycin, gentamicin and tetracycline; a DNA gyrase inhibitor levofloxacin; and an antimicrobial peptide nisin. Two strains (VR7 and MR7) resistant to various antimicrobial drugs were used in these experiments: MIC values of VRSA (VR7) and parental MRSA (MR7) against these antimicrobial drugs were higher than $64 \mu \mathrm{g} \mathrm{ml}^{-1}$ (Table 1). We determined the MIC values of vancomycin against VR7 in the presence of each antimicrobial drug $\left(20 \mu \mathrm{g} \mathrm{ml}^{-1}\right)$ that did not inhibit proliferation of the bacteria by itself. The results demonstrated that the MIC value of vancomycin against VR7 with $20 \mu \mathrm{g} \mathrm{ml}^{-1} \mathrm{D}$-cycloserine decreased to $1 / 4$ (from 16 to $4 \mu \mathrm{g} \mathrm{ml}^{-1}$; Table 2). On the other hand, the MIC value of vancomycin against VRSA was not decreased by bacitracin, fosfomycin, erythromycin, lincomycin, gentamicin, levofloxacin and nisin (Table 2). We further investigated the synergistic effects of vancomycin and D-cycloserine
Table 2 In vitro combinatorial effect of vancomycin and various antimicrobial drugs against VR7 and MR7

\begin{tabular}{lrrrrrrrrrrr}
\hline \multicolumn{1}{c}{ VAN } & VAN & VAN & VAN & VAN & VAN & VAN & VAN & VAN \\
\hline Strains & $V A N$ & $+C S$ & $+B C$ & $+F O M$ & $+E M$ & $+L C M$ & $+G M$ & + TC & + LVFX & $+N C$ \\
\hline VR7 & 16 & 4 & 16 & 16 & 16 & 16 & 16 & 8 & 16 & 16 \\
MR7 & 1 & 1 & 2 & 1 & 1 & 2 & 1 & 2 & 1 & 1 \\
\hline
\end{tabular}

Abbreviations: BC, bacitracin; CS, D-cycloserine; EM, erythromycin; FOM, fosfomycin;

GM, gentamicin; LCM, lincomycin; LVFX, levofloxacin; MIC, minimum inhibitory concentration; NC, nisin; TC, tetracycline; VAN, vancomycin.

MIC values of vancomycin in combination with each antimicrobial drug against VR7 or MR7 were determined by the micro-dilution method. The concentration of each antimicrobial drug used with vancomycin was $20 \mu \mathrm{g} \mathrm{ml}-1$. Unit: $\mu \mathrm{g} \mathrm{ml}^{-1}$

against seven other vancomycin-resistant strains (VR1-6, 8) that were independently isolated from each MRSA strain (MR1-6, 8). The MIC values of vancomycin against each strain with D-cycloserine at a $1 / 4$ concentration of the MIC were determined. We found that the MIC values of vancomycin for the seven strains were decreased in the presence of D-cycloserine. Especially, the MIC values of vancomycin against VR3, 6 and 7 decreased to $1 / 8$ (Table 3). The MIC value of vancomycin against VR8, which showed the smallest effect among the eight strains, decreased to $1 / 2$ (Table 3 ). We next determined the MIC values of vancomycin against VR7 in the presence of various concentrations of D-cycloserine. We found that the MIC value of vancomycin against VR7 by D-cycloserine was decreased at a concentration greater than $8 \mu \mathrm{g} \mathrm{ml}^{-1}$ D-cycloserine (Figure 1). As the MIC value of D-cycloserine against VR7 was $64 \mu \mathrm{g} \mathrm{ml}^{-1}$, the inhibitory effect of combined D-cycloserine and vancomycin on the growth of VR7 was synergistic when the concentration of D-cycloserine was $<20 \mu \mathrm{g} \mathrm{ml}^{-1}$.

Evaluation of the therapeutic effect of combined D-cycloserine and vancomycin in the silkworm VRSA infection model

We established systems to evaluate the therapeutic effects of combinations of antimicrobial drugs against VRSA using the silkworm infection model. ${ }^{8}$ In this study, we examined the therapeutic effect of combined vancomycin and D-cycloserine in a silkworm VRSA infection model. Vancomycin and D-cycloserine were administered to silkworms injected with VR7. The numbers of surviving silkworms were counted periodically. When vancomycin or D-cycloserine alone was injected, the silkworm survival period was not extended. On the other hand, combined injection of both drugs extended the silkworm survival period (Figure 2). All silkworms injected with saline survived $>65 \mathrm{~h}$ (data not shown). We therefore concluded that combined treatment with vancomycin and D-cycloserine was therapeutically effective against VRSA infection in silkworms. 
Table 3 In vitro combinatorial effect of vancomycin and D-cycloserine against eight VRSA strains and eight MRSA strains

\begin{tabular}{lrrrrrrr}
\hline Strains & CS & VAN & VAN +CS & Strains & CS & VAN & VAN +CS \\
\hline VR1 & 64 & 16 & $4^{\mathrm{a}}$ & MR1 & 128 & 2 & $1^{\mathrm{b}}$ \\
VR2 & 64 & 8 & $2^{\mathrm{a}}$ & MR2 & 64 & 2 & $2^{\mathrm{a}}$ \\
VR3 & 128 & 32 & $4^{\mathrm{b}}$ & MR3 & 256 & 2 & $2^{\mathrm{c}}$ \\
VR4 & 128 & 32 & $8^{\mathrm{b}}$ & MR4 & 64 & 2 & $1^{\mathrm{a}}$ \\
VR5 & 128 & 16 & $4^{\mathrm{b}}$ & MR5 & 64 & 2 & $2^{\mathrm{a}}$ \\
VR6 & 128 & 32 & $4^{\mathrm{b}}$ & MR6 & 64 & 2 & $1^{\mathrm{a}}$ \\
VR7 & 64 & 32 & $4^{\mathrm{a}}$ & MR7 & 128 & 1 & $1^{\mathrm{b}}$ \\
VR8 & 64 & 16 & $8^{\mathrm{a}}$ & MR8 & 64 & 2 & $2^{\mathrm{a}}$ \\
\hline
\end{tabular}

Abbreviations: CS, D-cycloserine; MIC, minimum inhibitory concentration; MRSA, methicillinresistant $S$. aureus; VAN, vancomycin; VRSA, vancomycin-highly resistant $S$. aureus. MIC values of CS, VAN and VAN in the presence of CS against VR7 and MR7 were determined by the micro-dilution method. The concentration of CS used with vancomycin was $1 / 4$ of the

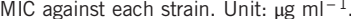

acS concentration was $16 \mu \mathrm{g} \mathrm{ml}-1$.

CS concentration was $32 \mathrm{ug} \mathrm{mt}^{-1}$.

c CS concentration was $64 \mu \mathrm{g} \mathrm{ml}-1$.

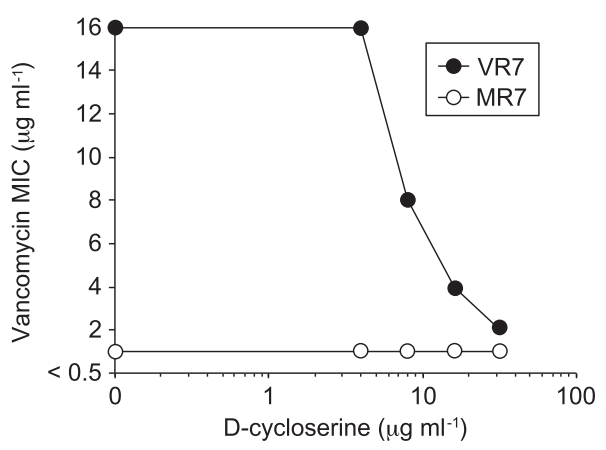

Figure 1 Titration of D-cycloserine in the combinatorial effect with vancomycin against VRSA obtained by mutagenesis. MIC values of vancomycin against VR7 or MR7 in the presence of each concentration of D-cycloserine $\left(0,4,8,16\right.$ and $\left.32 \mu \mathrm{g} \mathrm{ml}{ }^{-1}\right)$ were determined.

\section{DISCUSSION}

The findings of the present study demonstrated that D-cycloserine increased the susceptibility of VRSA strains to vancomycin. Moreover, vancomycin combined with D-cycloserine exhibited therapeutic effects in the silkworm VRSA infection model. This is a first report that D-cycloserine is synergistically effective with vancomycin against infectious disease caused by VRSA.

In the peptidoglycan biosynthesis of $S$. aureus, vancomycin inhibits the crosslinking of sugar chains by associating with D-alanine-D-alanine (D-Ala-D-Ala), which binds to a growing end of the GlcNac-MurNac chain. ${ }^{25}$ On the other hand, D-cycloserine inhibits the synthetic reaction of $\mathrm{D}-\mathrm{Ala}-\mathrm{D}-\mathrm{Ala}$ dipeptide from two molecules of $\mathrm{D}$-alanines, leading to a decrease in the supply of D-Ala-D-Ala, which is essential for peptidoglycan biosynthesis. ${ }^{26}$ Moreover, D-cycloserine increases the in vitro sensitivity of MRSA to daptomycin whose therapeutic mechanism involves the cell membrane and cell wall. ${ }^{27}$ We consider that a low concentration of D-cycloserine, which apparently does not exhibit antibacterial activity, might alter the structure of the cell surface to increase the susceptibility of bacteria to vancomycin. Further elucidation of the detailed molecular mechanism underlying the synergistic effects of vancomycin and D-cycloserine is a subject of future research.

We propose the administration of combinations of antibiotics whose clinical use was terminated due to the emergence of resistant

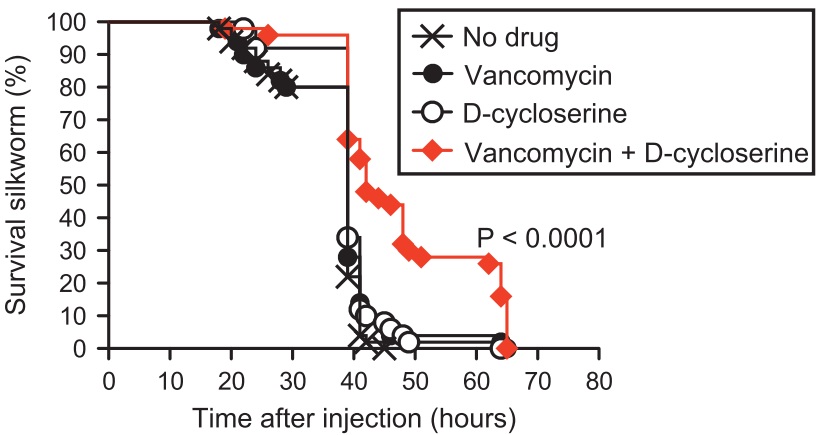

Figure 2 Therapeutic effect of combined D-cycloserine and vancomycin in silkworms infected by VRSA obtained by mutagenesis. Saline, vancomycin or D-cycloserine alone, or both vancomycin and D-cycloserine were injected into VR7-infected silkworms. Numbers of surviving silkworms were counted. Results of five independent experiments under the same conditions were assembled and analyzed. Statistical differences between each group were evaluated with the log-rank test. The $P$-value of the difference between vancomycin alone and combined vancomycin and D-cycloserine was less than 0.0001. The amounts of antimicrobial drugs injected were: vancomycin, $5 \mu \mathrm{g}$ per larva; D-cycloserine, $40 \mu \mathrm{g}$ per larva. The numbers of bacteria injected were: $0.45-1.1 \times 10^{8}$ c.f.u. per larva. $n=50$ per group.

bacteria as a general treatment strategy for drug-resistant bacterial infection. Abundant clinical data are available regarding the toxicity, pharmacokinetics and side effects of these antibiotics. We previously reported that the combination of a $\beta$-lactam and vancomycin was effective against a vancomycin-highly resistant strain. ${ }^{8}$ Based on these findings, we have proposed the concept 'drug re-use' for the treatment of infectious diseases. Here we demonstrated that the combination of D-cycloserine and vancomycin was also effective. These findings support the potential usefulness of 'drug re-use' as an approach toward developing effective treatments for infectious diseases caused by multidrug resistant bacteria.

As the genetic background of artificially generated VRSA strains used in this study is different to the clinical isolated VRSA strains that possess vanA gene, further studies will be required to evaluate the synergistic effects of vancomycin and D-cycloserine against the clinical isolated VRSA. As vancomycin-intermediate-resistant $S$. aureus strains are more frequently isolated in clinical infection than with VRSA strains, examination of efficacy of vancomycin in combination with D-cycloserine against vancomycin-intermediate-resistant $S$. aureus strains will be also important in future study. Moreover, elucidation of the effects using mouse infection models will be needed.

In conclusion, we found that D-cycloserine, a drug used mainly to treat tuberculosis patients, increased the effectiveness of vancomycin against VRSA. On the other hand, D-cycloserine has considerable toxicity when used at the doses to treat tuberculosis, but we expect that at lower doses where we have demonstrated synergy with vancomycin, D-cycloserine could be better tolerated. Further in vitro and in vivo studies will be required before clinical use for the treatment of resistant S.aureus infections.

\section{CONFLICT OF INTEREST}

KS has an advisory role at Genome Pharmaceuticals Institute Co., Ltd (Tokyo, Japan). All remaining authors declare no competing financial interests.

\section{ACKNOWLEDGEMENTS}

We thank Kana Hashimoto, Mari Maeda and Miki Takahashi (Genome Pharmaceuticals Institute Co., Ltd, Tokyo, Japan) for their technical assistance 
rearing the silkworms. This project was supported by JSPS KAKENHI grant number JP15H05783 (Scientific Research (S) to KS).

1 Howden, B. P., Davies, J. K., Johnson, P. D., Stinear, T. P. \& Grayson, M. L. Reduced vancomycin susceptibility in Staphylococcus aureus, including vancomycin-intermediate and heterogeneous vancomycin-intermediate strains: resistance mechanisms, laboratory detection, and clinical implications. Clin. Microbiol. Rev. 23, 99-139 (2010).

2 Chang, S. et al. Infection with vancomycin-resistant Staphylococcus aureus containing the vanA resistance gene. N. Engl. J. Med. 348, 1342-1347 (2003).

3 Melo-Cristino, J., Resina, C., Manuel, V., Lito, L. \& Ramirez, M. First case of infection with vancomycin-resistant Staphylococcus aureus in Europe. Lancet 382, 205 (2013).

4 Robert, J., Bismuth, R. \& Jarlier, V. Decreased susceptibility to glycopeptides in methicillin-resistant Staphylococcus aureus: a 20 year study in a large French teaching hospital, 1983-2002. J. Antimicrob. Chemother. 57, 506-510 (2006).

5 Wilson, P. et al. Linezolid resistance in clinical isolates of Staphylococcus aureus. J. Antimicrob. Chemother. 51, 186-188 (2003)

6 Bayer, A. S., Schneider, T. \& Sahl, H. G. Mechanisms of daptomycin resistance in Staphylococcus aureus: role of the cell membrane and cell wall. Ann. N. Y. Acad. Sci. 1277, 139-158 (2013).

7 Ishii, K. et al. Phenotypic and genomic comparisons of highly vancomycin-resistant Staphylococcus aureus strains developed from multiple clinical MRSA strains by in vitro mutagenesis. Sci. Rep. 5, 17092 (2015).

8 Tabuchi, F. et al. Synergistic effects of vancomycin and beta-lactams against vancomycin highly resistant Staphylococcus aureus. J. Antibiot. (Tokyo) (2017).

9 Brunetti, J., Falciani, C., Bracci, L. \& Pini, A. Models of in-vivo bacterial infections for the development of antimicrobial peptide-based drugs. Curr. Top. Med. Chem. 17, 613-619 (2016).

10 Krezdorn, J., Adams, S. \& Coote, P. J. A Galleria mellonella infection model reveals double and triple antibiotic combination therapies with enhanced efficacy versus a multidrug-resistant strain of Pseudomonas aeruginosa. J. Med. Microbiol. 63, 945-955 (2014).

11 Zhao, M., Lepak, A. J. \& Andes, D. R. Animal models in the pharmacokinetic/ pharmacodynamic evaluation of antimicrobial agents. Bioorg. Med. Chem. 24, 6390-6400 (2016).

12 Poulsen, M. O. et al. Combination therapy with thioridazine and dicloxacillin combats meticillin-resistant Staphylococcus aureus infection in Caenorhabditis elegans. J. Med. Microbiol. 63, 1174-1180 (2014).
13 Kaito, C., Akimitsu, N., Watanabe, H. \& Sekimizu, K. Silkworm larvae as an animal model of bacterial infection pathogenic to humans. Microb. Pathog. 32, 183-190 (2002).

14 Matsumoto, Y. et al. Quantitative evaluation of cryptococcal pathogenesis and antifungal drugs using a silkworm infection model with Cryptococcus neoformans. J. Appl. Microbiol. 112, 138-146 (2012).

15 Hamamoto, H. et al. Quantitative evaluation of the therapeutic effects of antibiotics using silkworms infected with human pathogenic microorganisms. Antimicrob. Agents Chemother. 48, 774-779 (2004).

16 Fujiyuki, T., Imamura, K., Hamamoto, H. \& Sekimizu, K. Evaluation of therapeutic effects and pharmacokinetics of antibacterial chromogenic agents in a silkworm model of Staphylococcus aureus infection. Drug Discov. Ther. 4, 349-354 (2010).

17 Usui, K. et al. Acute oral toxicity test of chemical compounds in silkworms. Drug Discov. Ther. 10, 57-61 (2016)

18 Hamamoto, $\mathrm{H}$. et al. Lysocin $\mathrm{E}$ is a new antibiotic that targets menaquinone in the bacterial membrane. Nat. Chem. Biol. 11, 127-133 (2015).

19 Uchida, R. et al. In vitro and in vivo anti-MRSA activities of nosokomycins. Drug Discov. Ther. 8, 249-254 (2014).

20 Uchida, R et al. Nosokomycins, new antibiotics discovered in an in vivo-mimic infection model using silkworm larvae. I: fermentation, isolation and biological properties. J. Antibiot. (Tokyo) 63, 151-155 (2010).

21 Tomoda, H. New approaches to drug discovery for combating MRSA. Chem. Pharm. Bull. (Tokyo) 64, 104-111 (2016).

22 Uchida, R., Iwatsuki, M., Kim, Y. P., Omura, S. \& Tomoda, H. Nosokomycins, new antibiotics discovered in an in vivo-mimic infection model using silkworm larvae. II: structure elucidation. J. Antibiot. (Tokyo) 63, 157-163 (2010).

23 Nakamura, I. et al. Discovery of a new antifungal agent ASP2397 using a silkworm model of Aspergillus fumigatus infection. J. Antibiot. (Tokyo) 70, 41-44 (2017).

24 Peng, H. L., Novick, R. P., Kreiswirth, B., Kornblum, J. \& Schlievert, P. Cloning, characterization, and sequencing of an accessory gene regulator (agr) in Staphylococcus aureus. J. Bacteriol. 170, 4365-4372 (1988).

25 Walsh, C. Molecular mechanisms that confer antibacterial drug resistance. Nature 406, 775-781 (2000).

26 Prosser, G. A. \& de Carvalho, L. P. Kinetic mechanism and inhibition of Mycobacterium tuberculosis D-alanine:D-alanine ligase by the antibiotic D-cycloserine. FEBS J. 280, 1150-1166 (2013)

27 Gasch, O. et al. Daptomycin in vitro activity against methicillin-resistant Staphylococcus aureus is enhanced by D-cycloserine in a mechanism associated with a decrease in cell surface charge. Antimicrob. Agents Chemother. 57, 4537-4539 (2013). 